\title{
Adecuación del protocolo de profilaxis antibiótica en las apendicectomías de población infantil
}

\author{
Adequacy of antibiotic prophylaxis protocol in appendectomies in children
}

\author{
Manuel Durán-Poveda1, Pablo Gil-Yonte², Diego Rodríguez-Villar ${ }^{3}$, Jesús García-Cruces, \\ Gil Rodríguez-Caravaca ${ }^{3,5 *}$ y Jesús San Román-Montero ${ }^{1}$ \\ ${ }^{1}$ Departamento de Medicina y Cirugía, Universidad Rey Juan Carlos, Madrid; '2Área de Cirugía General, Hospital Universitario Fundación Alcorcón, \\ Madrid; ${ }^{3}$ Departamento de Medicina Preventiva y Salud Pública, Universidad Rey Juan Carlos, Madrid; ${ }^{4}$ Hospital Universitario Río Carrión, Palencia; \\ ${ }^{5}$ Unidad de Medicina Preventiva, Hospital Universitario Fundación Alcorcón, Madrid. España
}

\begin{abstract}
Resumen
Objetivo: Evaluar la adecuación de la profilaxis antibiótica en la cirugía de apendicitis aguda en niños y su efecto en la infección del sitio quirúrgico. Método: Estudio de cohortes prospectivo para evaluar la adecuación al protocolo de la profilaxis antibiótica en apendicectomías en población infantil. Se evaluaron la administración de la profilaxis y las causas de la inadecuación. Se estudió el efecto de la inadecuación en la incidencia de infección del sitio quirúrgico con el riesgo relativo (RR) ajustado con un modelo de regresión logística por pasos hacia atrás. Resultados: Se estudiaron 412 pacientes. La profilaxis antibiótica estaba indicada en 348 pacientes y se administró en el 95.7\% de los casos, con una adecuación global al protocolo del 90.7\%. La causa principal del incumplimiento fue la hora de inicio. La incidencia acumulada de infección del sitio quirúrgico fue del 2.7\%. No se encontró relación entre la adecuación de la profilaxis y la infección del sitio quirúrgico (RR: 1.01; intervalo de confianza del 95\%: 0.95-1.11; $p=0.61$ ). Conclusiones: La adecuación de la profilaxis antibiótica fue alta, pero puede mejorarse. No se encontró relación entre la adecuación de la profilaxis antibiótica y la incidencia de infección del sitio quirúrgico.
\end{abstract}

PALABRAS CLAVE: Adecuación. Infección quirúrgica. Pediatría. Profilaxis antibiótica.

\begin{abstract}
Objective: To assess compliance of antibiotic prophylaxis in surgery for acute appendicitis in children and its effect on surgical site infection. Methods: We carried out a prospective cohort study to evaluate compliance of antibiotic prophylaxis in appendectomies in children. An assessment of the level of compliance with prophylaxis was made, as well as the causes of non-compliance. The effect of non-compliance of antibiotic prophylaxis on the incidence of surgical site infection was studied with the adjusted relative risk (RR) with a backstep logistic regression model. Results: The study included a total of 412 patients. Antibiotic prophylaxis was indicated in 348 patients, and administered in $95.7 \%$ of cases, with an overall protocol compliance of $90.7 \%$. The principal cause of non-compliance was time of initiation. Cumulative incidence of surgical site infection was 2.7\%. No relationship was found between inadequate prophylaxis compliance and infection (RR: 1.01; 95\% confidence interval: $0.95-1.11 ; p=0.61$ ). Conclusions: Compliance of antibiotic prophylaxis was high, but could be improved. No relationship was found between prophylaxis compliance and surgical site infection rate.
\end{abstract}

KEY WORDS: Antibiotic prophylaxis. Compliance. Pediatrics. Surgical wound infection.

\author{
Correspondencia: \\ *Gil Rodríguez Caravaca \\ Budapest, 1 \\ C.P. 28922, Alcorcón, Madrid, España \\ E-mail: grodriguez@fhalcorcon.es
}

Fecha de recepción: 14-08-2018

Fecha de aceptación: 30-01-2019

DOI:10.24875/CIRU.19000663
Cir Cir. 2019;87:410-415

Contents available at PubMed www.cirugiaycirujanos.com 


\section{Introducción}

La apendicectomía es el tratamiento habitual en la apendicitis aguda y se basa en la idea generalizada de que su curso evolutivo conduce a necrosis y perforación ${ }^{1}$. La infección de sitio quirúrgico es la complicación más frecuente de estos pacientes ${ }^{2}$ y su incidencia depende del grado de contaminación de la técnica quirúrgica y de determinados factores de riesgo intrínsecos o extrínsecos al paciente ${ }^{3}$. La infección del sitio quirúrgico aumenta el riesgo y la gravedad del paciente $e^{4,5} \mathrm{y}$ al ser la apendicectomía un procedimiento muy frecuente podemos evaluar su casuística, la incidencia de sus complicaciones y la calidad de la asistencia prestada ${ }^{6}$.

Los estudios actuales permiten recomendar, con un alto grado de evidencia, la profilaxis antibiótica previa a la cirugía como prevención de la infección del sitio quirúrgico. Ésta se produce por contaminación, durante el acto quirúrgico, del espacio intersticial, de los coágulos de fibrina o de los hematomas ${ }^{2,7}$. El objetivo principal de la profilaxis es conseguir unas concentraciones altas de antibiótico en el tejido durante el proceso quirúrgico y en las horas posteriores al cierre de la incisión. Si el antibiótico utilizado es suficientemente activo contra los posibles microorganismos contaminantes y se consiguen concentraciones tisulares de antibiótico elevadas durante todo el procedimiento quirúrgico, la profilaxis generalmente será eficaz ${ }^{8}$.

La mayoría de los estudios que evalúan el efecto de la profilaxis antibiótica en la incidencia de infección se han realizado en adultos y la evaluación de la adecuación de la profilaxis, y su efecto sobre la infección del sitio quirúrgico, es escasa en la población infantili ${ }^{9}$. Nuestro hospital cuenta con un protocolo de administración de profilaxis antibiótica (Tabla 1) que se actualiza de manera sistemática, cada dos años, según la evidencia disponible en la literatura. El objetivo de este estudio fue evaluar el grado de administración y adecuación de la profilaxis al protocolo en pacientes en edad infantil apendicectomizados y su efecto sobre la incidencia de infección del sitio quirúrgico.

\section{Método}

Se realizó un estudio de cohortes prospectivo. La evaluación se llevó a cabo en el Hospital Universitario Fundación Alcorcón (Madrid, España). El estudio incluyó pacientes del área de pediatría, de entre 3 y
16 años, intervenidos de apendicectomía en la unidad de cirugía general y del aparato digestivo desde el 1 de enero de 2008 hasta el 31 de diciembre de 2017.

Se hizo una estimación de tamaño muestral de 404 pacientes según un nivel de confianza del $95 \%$, una precisión del 5\%, una adecuación al protocolo mayor del $70 \%$ y unas pérdidas durante el seguimiento del $5 \%$. El estudio fue aprobado por el comité de ética e investigación clínica y por la comisión de investigación. Los pacientes fueron seleccionados mediante un proceso de inclusión consecutiva en el momento del diagnóstico de la apendicitis aguda. Las variables estudiadas fueron la edad, el sexo, el antibiótico, la dosis, la vía de administración, el tiempo de administración de la profilaxis, la duración de la administración, el tiempo de inicio y finalización de la cirugía, los procedimientos quirúrgicos de intervención del apéndice agrupados en el epígrafe APPY según los criterios de los Centers for Disease Control and Prevention (CDC) de los EE.UU. (Tabla 2), la existencia o ausencia de infección durante el periodo de seguimiento, el tipo de infección (incisional superficial, incisional profunda u órgano-espacio) y el microorganismo causante de la infección. Se usaron los criterios diagnósticos de los $C D{ }^{10}$ para el diagnóstico de infección del sitio quirúrgico. La identificación de los microorganismos causantes de las infecciones se hizo con el analizador MicroScan Walkaway (Siemens).

Se realizó un estudio descriptivo de la muestra describiendo las variables cualitativas con su distribución de frecuencias (número y porcentaje) y comparándolas con la prueba de ji al cuadrado de Pearson. Las variables cuantitativas se describieron con la media y la desviación estándar (DE). Se evaluó el criterio de normalidad con la prueba de Shapiro-Wilk y se comparó con la prueba no paramétrica de Mann-Whitney.

Se evaluó el porcentaje de administración de la profilaxis antibiótica y su adecuación comparándola con las pautas de administración indicadas en el protocolo en vigor en el hospital (Tabla 1). Se definió la administración adecuada de la profilaxis antibiótica como el cumplimiento de todos y cada uno de los criterios de administración descritos en el protocolo. Se estudiaron las siguientes causas de incumplimiento de la profilaxis: duración (prescripción y administración de más de una dosis), elección (antibiótico distinto al establecido en el protocolo), tiempo de inicio (administración más de una hora antes de la incisión), dosis (diferente de la definida en el protocolo) y vía de administración (no intravenosa). Puesto que el periodo máximo de incubación de una infección de la herida 
Cirugía y Cirujanos. 2019;87

Tabla 1. Protocolo de profilaxis antibiótica en la apendicectomía

\begin{tabular}{llcll}
\hline Profilaxis & Antibiótico & Dosis & Vía & Tiempo de administración \\
\hline Estándar & Amoxicilina-ácido clavulánico & $40 \mathrm{mg} / \mathrm{kg}(\mathrm{máximo} 2 \mathrm{~g})$ & Intravenosa & $30-60$ minutos previos a la cirugía \\
Pacientesalérgicos & Metronidazol+gentamicina & $500 \mathrm{mg}+3-5 \mathrm{mg} / \mathrm{kg}$ & Intravenosa & $30-60$ minutos previos a la cirugía \\
\hline
\end{tabular}

quirúrgica sin implantes es de 30 días $^{10}$, se estudió la incidencia de infección del sitio quirúrgico durante los 30 días siguientes a la cirugía, independientemente de si los pacientes permanecían ingresados o habían sido dados de alta. En los pacientes ingresados, la infección del sitio quirúrgico fue evaluada conjuntamente por un médico especialista en medicina preventiva, un pediatra y un cirujano. En los pacientes dados de alta se evaluó la infección de la herida en la consulta externa del hospital, en urgencias, en su centro de atención primaria (con la plataforma Horus de gestión de documentos de la historia clínica) o mediante una llamada telefónica si el paciente no había asistido a ninguno de estos centros asistenciales mencionados y no se encontraron registros en la historia de atención primaria. Se evaluó la relación entre la adecuación al protocolo de profilaxis antibiótica y la incidencia de infección del sitio quirúrgico con el riesgo relativo $(R R)$ ajustado por las diferentes covariables con un modelo de regresión logística por pasos hacia atrás.

Los datos se recogieron en una hoja diseñada ad hoc y se registraron en una base de datos estandarizada, normalizada y relacional diseñada en Access $^{\circledR}$ de Microsoft. El análisis estadístico se realizó con el programa SPSS 22 y se consideraron diferencias estadísticamente significativas aquellas con $p<0.05$.

\section{Resultados}

Se incluyeron en el estudio 412 pacientes; de ellos, $156(37.9 \%)$ eran niñas y $256(62.1 \%)$ eran niños. La edad media global fue de 11.5 años (DE: 2.9), con 11.4 años (DE: 3.1) para las mujeres y 11.7 años (DE: 2.8) para los hombres $(p=0.36)$. La mediana de edad global fue de 12 años (rango intercuartílico [RIQ]: 4), con una mediana de edad de 12 años para los niños (RIQ: 4) y de 11 años para las niñas (RIQ: 5). Solo 7 (1.7\%) pacientes tenían una edad de 3-4 años. La duración media de la intervención fue de $47.3 \mathrm{mi}$ nutos (DE: 24.5) y la mediana fue de 40.0 minutos (RIQ: 30). Los procedimientos quirúrgicos realizados fueron la apendicectomía abierta (84.5\%) y la apendicectomía laparoscópica (15.5\%).

La administración de profilaxis antibiótica estaba indicada en 348 (84.5\%) de los pacientes estudiados.
Tabla 2. Procedimientos quirúrgicos estudiados según la Clasificación Internacional de Enfermedades 9a Revisión, Modificación Clínica (CIE-9-MC)

\begin{tabular}{ll}
\hline CIE-9-MC & Procedimiento quirúrgico \\
\hline 47.01 & Apendicectomía laparoscópica \\
47.09 & Otra apendicectomía \\
47.2 & Drenaje de absceso apendicular \\
47.91 & Apendicostomía \\
47.92 & Cierre de fístula apendicular \\
47.99 & Otro procedimiento quirúrgico en el apéndice \\
\hline
\end{tabular}

Tabla 3. Adecuación a los diversos criterios de la profilaxis antibiótica $(n=333)$

\begin{tabular}{lccc}
\hline Criterio & $\begin{array}{c}\text { Cumplimiento } \\
\text { (n) }\end{array}$ & $\begin{array}{c}\text { Incumplimiento } \\
\text { (n) }\end{array}$ & $\begin{array}{c}\text { Adecuación } \\
\text { (\%) }\end{array}$ \\
\hline Duración & 332 & 1 & $99.7 \%$ \\
Vía & 333 & 0 & $100 \%$ \\
Antibiótico & 331 & 2 & $99.4 \%$ \\
Hora de inicio & 305 & 28 & $91.1 \%$ \\
Dosis & 333 & 0 & $100 \%$ \\
Total & 302 & 31 & $90.7 \%$ \\
\hline
\end{tabular}

El resto evolucionó con perforación y complicaciones que requirieron tratamiento antibiótico. La profilaxis se administró a 333 pacientes de los que la tenían indicada, lo que supone un porcentaje de cumplimiento de la administración del $95.7 \%$, y no pudo ser documentada en 15 (4.3\%) pacientes. Los antibióticos administrados fueron amoxicilina-ácido clavulánico $(97.3 \%)$, metronidazol-gentamicina $(1.0 \%)$, cefazolina $(1.0 \%)$, ceftriaxona $(0.4 \%)$ y ampicilina $(0.3 \%)$.

La adecuación global al protocolo de la profilaxis antibiótica, teniendo en cuenta todos los criterios de adecuación, fue del 90.7\%. La tabla 3 muestra el porcentaje y el total de pacientes que cumplieron cada criterio estudiado. La causa más frecuente de incumplimiento del protocolo fue la hora de inicio de la profilaxis, seguida por la elección del antibiótico.

La incidencia global de infección del sitio quirúrgico durante el seguimiento fue del 2.6\% (9 pacientes infectados), con una incidencia del $2.7 \%$ en la cirugía 
abierta y del $1.8 \%$ en la laparoscópica $(p=0.58)$. La incidencia acumulada de infección en el grupo de profilaxis adecuada fue del $1.6 \%$ y en el grupo de profilaxis inadecuada fue del $1.7 \%$. La densidad de incidencia de infección del sitio quirúrgico fue de 0.9 infecciones/1000 pacientes-día. Uno de los pacientes tuvo una infección polimicrobiana con dos microorganismos aislados en el exudado de la herida (Escherichia coli y Pseudomonas aeruginosa). El tipo de infección según la profundidad fue de un $88.9 \%$ de infección incisional superficial y un $11.1 \%$ de infección incisional profunda. La mayoría de los pacientes tuvieron un proceso de apendicitis sin complicaciones (88.6\%); el resto cursaron con apendicitis perforada y peritonitis $(9 \%)$ o con apendicitis y absceso apendicular $(2.4 \%)$. Los pacientes con complicaciones tuvieron más riesgo de cursar con infección del sitio quirúrgico (RR: 27.1; intervalo de confianza del 95\% [IC 95\%]: 5.8-127; $p<0.05$ ).

No encontramos relación entre la inadecuación de la profilaxis antibiótica y la incidencia de infección del sitio quirúrgico (RR: 1.01; IC 95\%: 0.95-1.11; $p=0.61$ ). El microorganismo más frecuente $(n=10)$ implicado en las infecciones quirúrgicas fue $E$. coli $(50 \%)$ (Fig. 1), y un paciente (10\%) con infección del sitio quirúrgico tuvo una infección polimicrobiana.

\section{Discusión}

Los protocolos hospitalarios en las apendicectomías en edad pediátrica contemplan la administración de profilaxis antibiótica previa a la cirugía, porque es una medida de eficacia probada para prevenir y reducir la frecuencia de infecciones del sitio quirúrgico $^{6}$. La incidencia de infección quirúrgica es un estándar de calidad en la atención sanitaria ${ }^{11}$ y hay estudios en los que se ha comunicado una reducción de la incidencia de infección del sitio quirúrgico de hasta un $56 \%{ }^{12}$ con la administración de profilaxis antibiótica.

Nuestro estudio evaluó el porcentaje de administración de profilaxis antibiótica en pacientes apendicectomizados y el grado de adecuación al protocolo hospitalario. El $95.7 \%$ de los pacientes recibieron tratamiento profiláctico, porcentaje menor que el observado en otras cirugías programadas ${ }^{13}$, lo que creemos que se debe a una descoordinación entre el momento del diagnóstico y el de la intervención quirúrgica, al tratarse de una cirugía de urgencia. Aun así, la adecuación en nuestra serie fue mayor que en otras de la literatura consultada sobre este

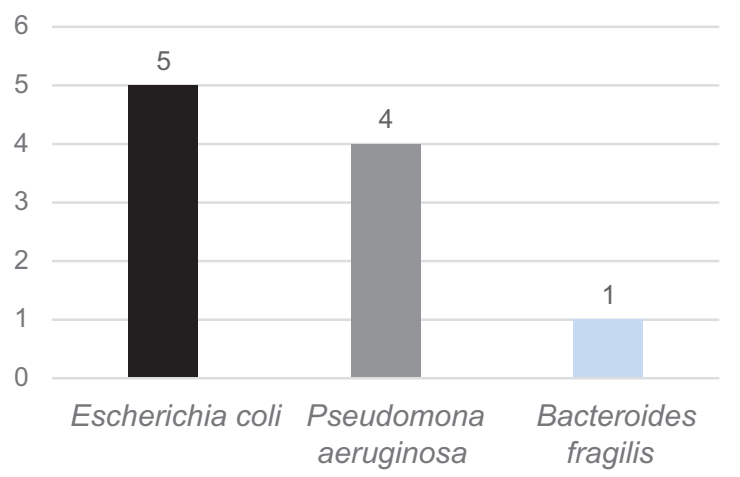

Figura 1. Etiología de la infección de sitio quirúrgico $(n=10)$.

procedimiento quirúrgico ${ }^{13-15}$. La administración de profilaxis no pudo ser constatada en el $4.3 \%$ de los pacientes debido a que no quedó constancia de ello en la historia clínica.

Entre los pacientes a quienes se administró profilaxis antibiótica, el porcentaje global de adecuación fue del $90.7 \%$, superior al de los estudios publicados consultados, tanto españoles ${ }^{16,17}$ como internacionales ${ }^{18,19}$. Los criterios individuales considerados (antibiótico administrado, vía, administración en la hora previa a la incisión, dosis y duración de la profilaxis) muestran grados desiguales de adecuación al protocolo, siendo la hora de inicio de la profilaxis (91.1\%) el criterio con menor porcentaje de adecuación. En otros estudios consultados, la duración de la profilaxis fue la que registró el mayor porcentaje de inadecuación ${ }^{16,20,21}$. Con respecto a la elección del antibiótico, el porcentaje de cumplimiento fue del $99.4 \%$, lo que supone un resultado muy positivo y que tiene poco margen de mejora. La adecuación en cuanto a la dosis y la vía de administración fue del $100 \%$.

La profilaxis antibiótica fue administrada por el personal de enfermería del quirófano y supervisada por los anestesiólogos, que son los médicos responsables de su administración de acuerdo con el protocolo. Las enfermeras y los anestesiólogos no sabían que iban a ser evaluados, por lo que se pudo controlar el efecto Hawthorne.

No encontramos relación significativa entre la adecuación de la profilaxis antibiótica y la incidencia de infección del sitio quirúrgico en los casos en que no se administró la profilaxis antibiótica, ni tampoco en aquellos en los que esta fue inadecuada. En caso de no administración de la profilaxis, la explicación más probable puede estar en que se trate de un fallo de registro y no realmente de su falta. En los pacientes con inadecuación de la hora de administración del antibiótico hay que tener en cuenta que las apendicectomías 
son intervenciones diagnosticadas en los servicios de urgencia y puede haber un retraso entre la valoración del paciente, la administración de la profilaxis y la realización del procedimiento quirúrgico ${ }^{22,23}$. Aun así, la duración de este tipo de cirugía es corta y la vida media del antibiótico permitiría una concentración bactericida en la incisión en el momento del procedimiento quirúrgico que podría explicarlo. Los pacientes con apendicitis complicada fueron los que más incidencia de infección del sitio quirúrgico presentaron, debido al incremento del riesgo de infección que la peritonitis o el absceso apendicular suponen ${ }^{24}$.

Respecto a los pacientes con profilaxis inadecuada debido a la elección del antibiótico, el número de casos es muy pequeño como para poder extraer conclusiones con una precisión adecuada. Sin embargo, es posible que, a pesar de no haber elegido el antibiótico definido en el protocolo, este haya sido eficaz contra la flora de nuestro hospital y no haber afectado a la incidencia de infección.

Las acciones dirigidas a la prevención de las infecciones son siempre unas medidas costo-efectivas ${ }^{25-29}$, lo cual, en un contexto de recursos limitados, debe verse como un valor añadido, tanto desde el punto de vista económico como desde el punto de vista de la mejora de la calidad de la atención y de la seguridad del paciente.

La fortaleza de este estudio radica en su carácter prospectivo y el alto número de apendicectomías analizadas, teniendo en cuenta que se trata de un estudio realizado en un hospital general y no en un hospital pediátrico. Este hecho influyó en que el número de pacientes de corta edad (3-4 años) fuera muy pequeño e insuficiente para evaluar el efecto de la profilaxis en este rango de edad. Puede ser una limitación de nuestro trabajo el hecho de que no se evaluara con la suficiente potencia estadística la relación entre la inadecuación de la profilaxis y la incidencia de infección. Nuestro objetivo fundamental fue evaluar el grado de adecuación de la profilaxis y en este sentido se estimó el tamaño muestral. Aun así, esta es una cohorte dinámica que nos permitirá hacer esa evaluación en un futuro próximo, cuando aumente el número de pacientes incluidos.

\section{Conclusiones}

La adecuación de la profilaxis antibiótica en la apendicectomía fue alta. La incidencia de infección del sitio quirúrgico fue baja y predominó en las apendicitis complicadas, pero deben realizarse todos los esfuerzos posibles para mejorarla. Es importante administrar la profilaxis antibiótica según los protocolos definidos y también evaluar su cumplimiento con el fin de tomar las medidas necesarias para mejorarla y reducir así la incidencia de infección del sitio quirúrgico. En este sentido, los programas de vigilancia y control de la infección son prioritarios.

\section{Agradecimientos}

Los autores agradecen a D. Sergio Rodríguez Villar su apoyo técnico en el registro y la gestión de los datos.

\section{Conflicto de intereses}

Los autores declaran no tener ningún conflicto de intereses.

\section{Financiación}

Los autores agradecen al Instituto de Salud Carlos III y a los Fondos Europeos para el Desarrollo Regional (FEDER) la financiación de este estudio con los proyectos de investigación números PI11/01272 y PI14/01136.

\section{Responsabilidades éticas}

Protección de personas y animales. Los autores declaran que los procedimientos seguidos se conformaron a las normas éticas del comité de experimentación humana responsable y de acuerdo con la Asociación Médica Mundial y la Declaración de Helsinki.

Confidencialidad de los datos. Los autores declaran que han seguido los protocolos de su centro de trabajo sobre la publicación de datos de pacientes.

Derecho a la privacidad y consentimiento informado. Los autores declaran que en este artículo no aparecen datos de pacientes.

\section{Bibliografía}

1. Gorter RR, The SML, Gorter-Stam MAW, Eker HH, Bakx R, van der Lee $\mathrm{JH}$, et al. Systematic review of nonoperative versus operative treatment of uncomplicated appendicitis. J Pediatr Surg. 2017;52:1219-27.

2. Lee SL, Islam S, Cassidy LD, Abdullah F, Arca MJ; 2010 American Pediatric Surgical Association Outcomes and Clinical Trials Committee. Antibiotics and appendicitis in the pediatric population: an American Pediatric Surgical Association Outcomes and Clinical Trials Committee systematic review. J Pediatr Surg. 2010;45:2181-5.

3. Sax H, Uçkay I, Balmelli C, Bernasconi E, Boubaker K, Mühlemann K, et al. Overall burden of healthcare-associated infections among surgical patients. Results of a national study. Ann Surg. 2011;253:365-70.

4. Edwards JR, Peterson KD, Mu Y, Banerjee S, Allen-Bridson K, Morrell G, et al. National Healthcare Safety Network (NHSN) report: data summary 


\section{Durán-Poveda, et al.: Profilaxis antibiótica en apendicectomías}

for 2006 through 2008, issued December 2009. Am J Infect Control. 2009;37:783-805

5. Kirkland KB, Bridges JP, Trivette SL, Kirkland KB, Briggs JP, Trivette SL, et al. The impact of surgical site infections in the 1990s: attributable mortality, excess length of hospitalization, and extra costs. Infect Control Hosp Epidemiol. 1999;20:725-30.

6. Rogers AP, Zens TJ, Leys CM, Nichol PF, Ostlie DJ. A call for a standardized definition of perforated appendicitis. J Pediatr Surg. 2017:52:89-92.

7. Sandora TJ, Fung M, Melvin P, Graham DA, Rangel SJ. National variability and appropriateness of surgical antibiotic prophylaxis in US children's hospitals. JAMA Pediatr. 2016;170:570-6.

8. Andersen BR, Kallehave FL, Andersen HK. Antibiotics versus placebo for prevention of postoperative infection after appendicectomy. Cochrane Database Syst Rev. 2005;(20):CD001439.

9. Mueck KM, Putnam LR, Anderson KT, Lally KP, Tsao K, Kao LS. Does compliance with antibiotic prophylaxis in pediatric simple appendicitis matter? J Surg Res. 2017;216:1-8.

10. Horan T, Andrus M, Dudeck M. CDC/NHSN surveillance definition of health care-associated infection and criteria for specific types of infections in the acute care setting. Am J Infect Control. 2008;36:309-32.

11. Dellinger EP, Gross PA, Barret TL, Krause PJ, Martone WJ McGowan JE Jr, et al. Quality standard for antimicrobial prophylaxis in surgical procedures. Clin Infect Dis. 1994;18:422-7.

12. Martin C; the French Study Group on Antimicrobial Prophylaxis in Surgery. Antimicrobial prophylaxis in surgery: general concepts and clinical guidelines. Infect Control Hosp Epidemiol. 1994:15:463-71.

13. Sánchez-Santana T, Del-Moral-Luque JA, Gil-Yonte P, Bañuelos-Andrío L, Durán-Poveda M, Rodríguez-Caravaca G. Effect of compliance with an antibiotic prophylaxis protocol in surgical site infections in appendectomies. Prospective cohort study. Cir Cir. 2017;85:208-13.

14. Kasatpibal N, Jamulitrat S, Chongsuvivatwong V. Standardized incidence rates of surgical site infection: a multicenter study in Thailand. Am J Infect Control. 2005;33:587-94.

15. Jodrá VM, Díaz-Agero Pérez C, Sainz de los Terreros Soler L, Saa Requejo CM, Dacosta Ballesteros D. Results of the Spanish national nosocomial infection surveillance network (VICONOS) for surgery patients from January 1997 through December 2003. Am J Infect Control. 2006;34:134-41.

16. Del-Moral-Luque JA, Colás-Ruiz E, Gil-Yonte $P$, Fernández-Cebrián JM, Villar-Del-Campo MC, Delgado-Iribarren A, et al. Assessment of antibiotic prophylaxis adequacy in rectal surgery. Rev Esp Quimioter. 2017; 30:14-1.

17. Garrote-Garrote M, Del-Moral-Luque JA, Checa-García A, Valverde-Cánovas JF, Campelo-Gutiérrez C, Martínez-Martín J, et al. Prophylactic antibiotherapy in hip arthroplasty. Cohort study. Rev Esp Quimioter. 2018; 31:118-22.

18. Forbes S, Stephen WJ, Harper WL, Loeb M, Smith R, Christoffersen EP, et al. Implementation of evidence-based practices for surgical site infection prophylaxis: Results of a pre and postintervention study. J Am Coll Surg. 2008;207:336-41.

19. Fennessy BG, O'Sullivan MJ, Fulton GJ, Kirwan WO, Redmond HP. Prospective study of use of perioperative antimicrobial therapy in general surgery. Surg Infect (Larchmt). 2006;7:355-60.

20. Díaz-Agero-Pérez C, Pita-López MJ, Robustillo-Rodela A, Figuerola-Tejerina A, Monge-Jodrá V. Evaluación de la infección de herida quirúrgica en 14 hospitales de la Comunidad de Madrid: estudio de incidencia. Enferm Infecc Microbiol Clin. 2011;29:257-62.

21 Del-Moral-Luque JA, Checa-García A, López-Hualda A, Villar-Del-Campo MC, Martínez-Martín J, Moreno-Coronas FJ, et al. Antibiotic prophylaxis adequacy in knee arthroplasty and surgical wound infection: prospective cohort study. Rev Esp Cir Ortop Traumatol. 2017:61:259-64.

22 Litz CN, Asuncion JB, Danielson PD, Chandler NM. Timing of antimicrobial prophylaxis and infectious complications in pediatric patients undergoing appendectomy. J Pediatr Surg. 2018;53:449-51.

23. Burke JF. The effective period of preventive antibiotic action in experimental incisions and thermal lesions. Surgery. 1961;50:161-8.

24 Hamid KA Mohamed MA, Salih A. Acute appendicitis in young children: a persistent diagnostic challenge for clinicians. Cureus. 2018;10:e2347.

25. Geubbels EL, Bakker HG, Houtman P, van Noort-Klaassen MA, Pelk MS, Sassen TM, et al. Promoting quality through surveillance of surgical site infections: five prevention success stories. Am J Infect Control. 2004; 32:424-30.

26. Kao LS, Lew DF, Doyle PD, Carrick MM, Jordan VS, Thomas EJ, et al. A tale of 2 hospitals: a staggered cohort study of targeted interventions to improve compliance with antibiotic prophylaxis guidelines. Surgery. 2010;148:255-62.

27. Yin Y, Song T, Liao B, Luo Q, Zhou Z. Antibiotic prophylaxis in patients undergoing open mesh repair of inguinal hernia: a meta-analysis. Am Surg. 2012;78:359-65.

28. Hedrick TL, Turrentine FE, Smith RL, McElearney ST, Evans HL, Pruett $T L$, et al. Single-institutional experience with the surgical infection prevention project in intra-abdominal surgery. Surg Infect (Larchmt). 2007:8:425-35.

29. Cárdenas-Salomon CM, Cervantes-Castro J, Jean-Silver ER, Toledo-Valdovinos SA, Murillo-Zolezzi A, Posada-Torres JA. Hospitalization costs of open vs. laparoscopic appendectomy: 5-year experience. Cir Cir. 2011;79:534-9. 